\title{
Evaluation of Factors Influencing Lymph Node Metastasis in Grade 3 Endometrial Cancers: A Retrospective Study
}

\author{
Bo Wang ${ }^{1}$, Qian Wang ${ }^{1}$, Yue $\mathrm{Shi}^{1}$, Wenyu Shao ${ }^{1}$, Jiongbo Liao ${ }^{1}$, Xue-zhen Luo ${ }^{1}$, Xiaojun \\ Chen $^{1}$, and Chao Wang ${ }^{1}$ \\ ${ }^{1}$ Obstetrics and Gynecology Hospital of Fudan University
}

July 16, 2020

\begin{abstract}
Objective: To evaluate the risk of lymph node metastasis and figure out necessity of lymphadenectomy in grade 3 endometroid endometrial cancer (EEC) patients. Methods: From 2009 to 2019, 3751 endometrial cancer (EC) patients were diagnosed at Gynecology Hospital of Fudan University, 1235 EEC patients were enrolled in multivariable analysis. 381 patients had survival analysis attributed to sufficient follow-up information. Kaplan-Meier curve and logrank test were used to analyze the survival rate. Results: Among 1235 EEC patients, multivariate analysis showed lymph-vascular invasion (LVSI), adnexal involvement (AI), cervical stroma involvement (CSI) were independent risk factors of lymph node metastasis (LNM) in grade 3 (G3) cohort $(\mathrm{OR}=3.45,5.83,8.93 ; 95 \%$ CI 1.12-10.64, 1.52-22.35, 2.85-28.00 respectively). LNM rates increased from $3.3 \%$ to $75 \%$ for EEC G3 cohort with the increase of risk factors from one to three. There were no differences between G3 and grade 1\&2 (G1\&2) in overall survival or progression free survival. Similarly, no survival advantage was found for EEC G3 patients at early stage with different plans of adjuvant treatment. Conclusion: When EEC G3 patients combine with one or more risk factors including LVSI, AI and CSI, lymphadenectomy is recommended. For those with only one factor of G3, it is recommended to evaluate the status of lymph nodes by considering other methods such as sentinel lymph node biopsy technology to avoid overtreatment. Survival analysis showed no difference in EEC G3 cohort compared with G1\&2. Also, different plans of adjuvant treatment had no impact on overall survival for EEC G3 patients.
\end{abstract}

Evaluation of Factors Influencing Lymph Node Metastasis in Grade 3 Endometrial Cancers: A Retrospective Study

Bo Wang ${ }^{1,2}$ \#, Qian Wang ${ }^{1,2}$ \#, Yue Shi ${ }^{1,2}$,Wenyu Shao ${ }^{1,2}$, Jiongbo Liao $^{1,2}$, Xuezhen Luo ${ }^{1,2}$, Xiaojun Chen ${ }^{1,2} *$,Chao Wang ${ }^{1,2} *$

1. Obstetrics and Gynecology Hospital, Fudan University, Shanghai 200011, People's Republic of China

2. Shanghai Key Laboratory of Female Reproductive Endocrine Related Diseases, Shanghai 200011, people's Republic of China

\# These authors contributed equally to this work.

Address correspondence to:

Xiaojun Chen, Department of Obstetrics and Gynecology, Obstetrics and Gynecology Hospital, Fudan University. 419 Fang Xie Rd., Huang Pu District, Shanghai, 200011, China. Phone: +86 13601680784. Email:13601680784@163.com.

Chao Wang, Department of Obstetrics and Gynecology, Obstetrics and Gynecology Hospital, Fudan University. 419 Fang Xie Rd., Huang Pu District, Shanghai, 200011, China. Phone: +86 13816070618. Email:wang1980-55@163.com. 
Objective: To evaluate the risk of lymph node metastasis and figure out necessity of lymphadenectomy in grade 3 endometroid endometrial cancer (EEC) patients.

Methods: From 2009 to 2019, 3751 endometrial cancer (EC) patients were diagnosed at Gynecology Hospital of Fudan University, 1235 EEC patients were enrolled in multivariable analysis. 381 patients had survival analysis attributed to sufficient follow-up information. Kaplan-Meier curve and logrank test were used to analyze the survival rate.

Results: Among 1235 EEC patients, multivariate analysis showed lymph-vascular invasion (LVSI), adnexal involvement (AI), cervical stroma involvement (CSI) were independent risk factors of lymph node metastasis (LNM) in grade $3(\mathrm{G} 3)$ cohort ( $\mathrm{OR}=3.45,5.83,8.93 ; 95 \%$ CI 1.12-10.64, 1.52-22.35, 2.85-28.00 respectively). LNM rates increased from $3.3 \%$ to $75 \%$ for EEC G3 cohort with the increase of risk factors from one to three. There were no differences between G3 and grade $1 \& 2$ (G1\&2) in overall survival or progression free survival. Similarly, no survival advantage was found for EEC G3 patients at early stage with different plans of adjuvant treatment.

Conclusion: When EEC G3 patients combine with one or more risk factors including LVSI, AI and CSI, lymphadenectomy is recommended. For those with only one factor of G3, it is recommended to evaluate the status of lymph nodes by considering other methods such as sentinel lymph node biopsy technology to avoid overtreatment. Survival analysis showed no difference in EEC G3 cohort compared with G1\&2. Also, different plans of adjuvant treatment had no impact on overall survival for EEC G3 patients.

\section{Key words}

Endometrioid endometrial cancer; Grade 3; Lymph node metastasis; Risk factors; Prognosis

\section{Highlights}

Lymph-vascular space invasion, adnexal involvement, cervical stroma involvement are independent risk factors for grade 3 endometroid endometrial cancer patients.

And the lymph node metastasis rates of grade 3 patients might increase with these risk factors.

There is no survival difference in grade 3 cohorts with different adjuvant treatment.

\section{Introduction}

Endometrial cancer (EC) is the fifth most common gynecological malignancy and the prevalence is increasing ${ }^{1 .} 569847$ new cases of EC were diagnosed in 2018 worldwide $^{2}$, and the number of EC patients would increase to $42.13 / 100000$ patients in 2030 in USA as prediction ${ }^{3}$. In China, the incidence of EC had an upward trend for decades ${ }^{4}$. Around $75 \%$ of EC patients were diagnosed at an early stage (FIGO stage I or II) and could be treated timely, therefore EC had a relatively good 5-year overall survival (OS) ranged from $74 \%$ to $91 \%{ }^{4,5}$. The main treatment of EC is standard surgery (including hysterectomy, bilateral salpingo-oophorectomy and evaluation of lymph node metastasis). Risk factors of EC are often taken into consideration for the decision of the operation extent.

Grade 3 had been classified as one of the high-risk factors for recommending comprehensive surgery including pelvic or para aortic lymphadenectomy (LND) as to Mayo Clinic standard ${ }^{6,7}$. Can we perform systemic lymphadenectomy for every G3 EC patient?

Some researchers suggested treatments like exclusive LND are not always necessary. Two randomized trials evaluated systematic pelvic LND compared with no LND and neither trial showed survival benefit for the LND arm in early EC patients ${ }^{8,9}$. For avoiding excessive side effects of LND, the biopsy of sentinel lymph node (SLN) has been used in the high-risk type of endometrial cancer recently ${ }^{10,11}$, but this technique is not widely used, especially in regions with poor medical resource. In addition, there is also an awkward situation of pathological upgrading to G3 only after the operation. Therefore, it is a challenge to make a decision of surgical spectrum before operations and achieve the goal of maximum resection of lesions and minimum injury. 
this retrospective analysis was done to investigate the relevant risk factors for lymph node metastasis (LNM) in EEC G3 patients so that better clinical decisions can be made to avoid overtreatment, especially in hospitals without sentinel biopsy techniques.

\section{Methods}

3751 patients with endometrial cancer diagnosed in Obstetrics and Gynecology Hospital of Fudan University were enrolled from Jan. 2009 to April. 2019. patients who did not receive comprehensive staging surgery $(\mathrm{n}=2516)$, with unknown/inconsistent pathologic staging $(\mathrm{n}=80)$, or unknown endometrioid histology type $(\mathrm{n}=331)$ were excluded. Finally, 1235 EEC patients were analyzed to hunt for the significant clinicopathological risk factors. 854 patients were excluded for survival analysis due to missing follow-up information, therefore, only 381 patients were included for survival analysis from Jan. 2009 to June. 2018.

Among 1235 EEC patients, univariable and multivariable analysis were done for risk factor analysis. These factors included age, Endo, Microcystic, Elongated, and Fragmented (MELF) pattern of invasion, stage, lymph vascular space invasion (LVSI), cervical stroma involvement (CSI), adnexal involvement (AI), parametrium involvement (PI), tumor size (TS), and myometrium invasion (MI). Then we stratified risk factors which strongly related to lymph node LNM rates in grade 3 patients.

The primary end-point was defined as death, overall survival (OS) was defined as the time from the date of diagnosis until death or last follow-up till June. 2018. The secondary endpoint was endometrial cancer recurrence, and progression-free survival (PFS) estimates were also assessed. Medical records were reviewed to determine OS and PFS according to the status of lymph node metastasis.

Variables were evaluated by Pearson $\mathrm{Chi}^{2}$ test and Yates' adjusted $\mathrm{Chi}^{2}$ test. Binary regression models were used for multivariate analysis. survival outcomes were indicated with OS and PFS. While survival rates were estimated using Kaplan-Meier method, the log-rank test was utilized to draw survival curve. Statistical analysis was performed with STATA 15.0 and Prism 8.

\section{Results}

Characteristics of study cohort

1235 cases were analyzed according to the inclusion criteria, including 181 cases of grade $3(14.7 \%), 1054$ cases of grade $1 \& 2(85.3 \%)$. For survival analysis, 53 grade 3 patients $(13.9 \%)$ and 328 grade $1 \& 2$ patients (86.1\%) were included respectively (Figure1).

The LNM rate $(16.6 \%)$ in overall grade 3 population is around twice higher than that of grade $1 \& 2(9.4 \%)$. patients over 70 years old in grade $1 \& 2$ group presented with highest LNM rate. There is no obvious difference among these age subgroups of grade 3. EEC G3 patients with any of the following risk factors including LVSI, CSI, AI, or MI, the risk of LNM would increase by 3 to 5 folds (Table 1).

After adjusted for age, parametrium involvement and tumor size in multivariate analysis, we found LVSI, $\mathrm{AI}$ and CSI were independent risk factors for LNM (Adjusted OR=3.45, 5.83, 8.93; 95\% CI 1.12-10.64, 1.52-22.35, 2.85-28.00 respectively) in EEC G3 cohort. LVSI, AI, MELF, MI (Adjusted OR=8.82, 3.40, 2.24, 5.02 ; $95 \%$ CI 4.88-15.96, 1.29-8.95, 1.28-3.93, 2.93-8.61, respectively) were independent risk factors among grade $1 \& 2$ cohort (Table 3 ). The overall population presented with similar trends with grade $1 \& 2$ subgroup (Adjusted OR=6.95, 3.21, 2.67, 4.30;95\% CI 4.12-11.72, 1.51-6.81, 1.56-4.54, 2.66-6.94, respectively) (Table 2 ). For our data, we observed tumor size increased the risk of LNM for grade $1 \& 2$ other than grade 3 .

Stratification analysis for risk factors in lymph node metastasis

Among 181 EEC G3 patients, the general LNM rate was 16.7\% (30 out of 181). With negative LVSI, CSI and $\mathrm{AI}$, the LNM rate was $3.3 \%(3 / 92)$. When combined with single positive LVSI or CSI or AI, the LNM rate was $14.5 \%(8 / 55)$ or $28.6 \%$ (2/7) or $50.0 \%(1 / 2)$ respectively. The LNM rate increased to $75.0 \%(9 / 12)$ when combined with both positive LVSI and CSI. Similarly, with positive LVSI and AI, the LNM rate reached to $60.0 \%(6 / 10)$. LNM rate of EEC G3 patients with positive LVSI, CSI and AI was 33.3\% (1/3) (Table 4). 


\section{Survival analysis}

From Jan. 2009 to June 2018, no patient died from endometrial cancer with follow-up, 7 (1.8\%) patients had recurrence (Table 5). 5-year overall survival was $100.0 \%$ and 5-year progression survival was $98.2 \%$ (7/381). Kaplan-Meier curves showed grade 3 patients were not associated with shorter overall survival or progression free survival compared with grade $1 \& 2$ group (logrank p $>0.05$ ) (Figure 2). In EEC G3 patients at stage I or II, overall survival and progression free survival analysis showed no distinct difference of two subgroups: The first was divided into chemotherapy, radiotherapy and chemoradiotherapy (log rank p $>0.05$ ); the second was classified as pelvic LND (PVLND) and pelvic + para aortic LND (PV+PALND) (log rank p >0.05) (Figure $3)$.

\section{Discussion}

Age, histological grade, myometrial invasion (MI), lymph-vascular space invasion (LVSI), tumor size (TS) have been proved to have value on prediction of lymph node metastasis (LNM) which might help physicians to make better clinical decisions ${ }^{12-15}$. How can we achieve the goal of maximum resection of lesions and cause minimum injury if it is not accessible to use SLN. We believe the pathologic grade should be the most reliable and accessible factor. That's the reason grade 3 was chosen as the indicator to evaluate the possibility of LNM in this study.

Inconsistent standard were published by different institutions: Mayo and FIGO(The International Federation of Gynecology and Obstetrics) guidelines tend to categorize TS $>2 \mathrm{~cm}$ as high-risk, while for NCCN (The National Comprehensive Cancer Network) and ESMO (Europe's leading medical oncology society) guidelines, TS was not considered as a high-risk factor ${ }^{5,16,17}$. In fact, it is uneasy to determinate the size of tumor due to its irregular shape of lesion, and the latest NCCN guideline had deleted this factor ${ }^{16}$.

It is true that deep myometrial invasion increased the risk of lymph nodes metastasis based on suggestions of almost every international or national guideline and our data (not shown in this study). However, it is not easy to accurately evaluate the deep myometrial invasion before operation. Although the NCCN guidelines recommend the use of pelvic enhanced MRI to determine the depth of myometrial invasion (myoinvasion $>50 \%$ is considered as a risk factor $)^{16}$, the accuracy of MRI is about $68 \%$ for T2-weithgted imaging ${ }^{18}$.

For age, it was considered older age was associated with higher chance of LNM, while the age cut-off was still inconsistent. According to the latest NCCN guidelines, "age $>=60$ " was considered as an age cut-off ${ }^{16}$, while in our data $>=70$-year-old seemed to have significant impact on LNM.

For LVSI, never to be ignored in EC carcinogenesis, which has been emphasized again recently. LVSI is a potential predictor for EC recurrence ${ }^{14,19}$ and associated with a significant higher rate of paraaortic LNM with OR at five ${ }^{20}$. A retrospective study showed no overall survival difference between adjuvant external beam radiation therapy versus vaginal brachytherapy in LVSI-positive patients, while it was associated with increased risk of death (hazard ratio of 1.94) ${ }^{21}$. Unfortunately, it is quite difficult to get before operation.

Finally, we selected G3 as our first concern in making decisions before operations of EC patients. We found that EEC G3 patients without any extra risk factor (LVSI, AI or CSI) had an LNM rate of 16.4\%, which is remarkably lower than other risk factors, for which LND might not be needed. On the contrary, EEC G3 patients with one or more extra risk factors mentioned above had higher chances of lymph node metastasis, which could be explained by synergetic effects in between these risk factors. Grade 3 EEC patients with only one risk factor presented with an LNM rate ranged at $14.5 \%$ to $50 \%$ while if with two extra risk factors, LNM rate increased to $60 \%$ and $75 \%$. Although grade 3 EEC patients with positive LVSI, AI and CSI, was found to have lower LMN incidence at 33.3\%, also known as 1 out of 3 . We assume it was due to the limited sample size.

The categorization of three tiers of LVSI was mentioned by pathologists to evaluate its potential risk ${ }^{19}$. Although our study failed to categorize LVSI into three tiers, our findings are consistent with these opinions. LVSI were independent risk factors of both grade 3 and grade $1 \& 2$ subgroups, increased the LNM rates more than 3 folds and 8 folds, respectively. Therefore, we recommend EEC G3 patients with LVSI should 
undertake lymph node evaluation, EEC G3 patients with no other risk factor could be evaluated only with imaging, while EEC G3 patients with positive LVSI in their final pathological diagnosis, should be evaluated by both imaging and comprehensive staging surgery if not done previously, which is consistent with the latest NCCN guidelines ${ }^{16}$.

Two randomized trials indicated that systematic pelvic LND had no survival benefit compared with no LND in early EC patients ${ }^{8,9}$. Our data showed no survival difference between grade 3 and grade $1 \& 2$ patients, which indicated that "grade" might not influence patients' prognosis although grade 3 patients had higher rate of LNM than grade $1 \& 2$ patients. This could be due to the small sample size of grade 3 patients. Similarly, patients with pelvic and para-aortic lymph node dissection done had similar OS and PFS than patients with only pelvic lymph node dissection done in grade 3 population at early stage, suggesting a less traumatic treatment could be considered in these patients.

In addition, we found that MELF and MI only increased the LNM rate of grade $1 \& 2$ population, while CSI increased the LNM risk of grade 3 patients. This might indicate different metastasis pathways in EC patients with different grades, for which further investigations should be carried out.

In this study, our strength stood on the follow-up duration of almost ten years. In addition, the independent high-risk factors were robust with adjustments. We also provided clinically friendly data by stratifying these risk factors, providing certain evidence for clinical practice especially in areas with poor medical resource. However, this study still had some limitations. Since this is a retrospective analysis, selection bias may have influenced the treatments given to these grade 3 patients. Only 381 (30.9\%) patients were found to have complete follow-up data due to technical reasons. Larger sample size and prospective clinical trial design with novel molecular classification warrant more consideration.

\section{FUNDING SUPPORT}

This work was funded by institutional support from Shanghai Science and technology committee medical guidance program (18411963700), "Shanghai Pujiang Program"(17PJ1401400), National Natural Science Foundation of China(81772777).

\section{CONFLICT OF INTEREST DISCLOSURES}

The authors declare no conflicts of interest to disclose.

\section{AUTHOR CONTRIBUTIONS}

Bo Wang and Qian Wang: Writing- Original draft preparation. Yue Shi: Software, Validation. Wenyu Shao and Jiongbo Liao: Validation. Xuezhen Luo and Xiaojun Chen: Reviewing and Editing. Chao Wang: Conceptualization, Methodology, Supervision.

\section{ACKNOWLEDGMENTS}

None.

\section{ETHICS APPROVAL AND CONSENT TO PARTICIPATE}

This study partly used already published data from anonymized patients (doi: 10.1371/journal.pone.0155145). Ethical approval and consent to participate were granted for original studies by the Ethics Committee of the Obstetrics and Gynecology Hospital of Fudan University. Our research based on different topic so the another approval is on the process.

\section{REFERENCES}

1. Siegel RL, Miller KD, Jemal A. Cancer statistics, 2019. CA Cancer J Clin. Jan 2019;69(1):7-34.

2. Bray F, Ferlay J, Soerjomataram I, Siegel RL, Torre LA, Jemal A. Global cancer statistics 2018: GLOBOCAN estimates of incidence and mortality worldwide for 36 cancers in 185 countries. Ca-Cancer J Clin. Nov-Dec 2018;68(6):394-424. 
3. Sheikh MA, Althouse AD, Freese KE, et al. USA Endometrial Cancer Projections to 2030: should we be concerned? Future Oncol.2014;10(16):2561-2568.

4. Chen W, Zheng R, Baade PD, et al. Cancer statistics in China, 2015. CA: a cancer journal for clinicians.2016;66(2):115-132.

5. Creasman WT, Odicino F, Maisonneuve P, et al. Carcinoma of the corpus uteri. FIGO 26th Annual Report on the Results of Treatment in Gynecological Cancer. Int J Gynaecol Obstet. 2006;95 Suppl 1:S105S143.

6. Kumar S, Podratz KC, Bakkum-Gamez JN, et al. Prospective assessment of the prevalence of pelvic, paraaortic and high paraaortic lymph node metastasis in endometrial cancer. Gynecol Oncol.2014;132(1):3843.

7. Mariani A, Dowdy SC, Cliby WA, et al. Prospective assessment of lymphatic dissemination in endometrial cancer: a paradigm shift in surgical staging. Gynecol Oncol. 2008;109(1):11-18.

8. Panici PB, Basile S, Maneschi F, et al. Systematic Pelvic Lymphadenectomy vs No Lymphadenectomy in Early-Stage Endometrial Carcinoma: Randomized Clinical Trial. J Natl Cancer I. Dec 3 2008;100(23):17071716 .

9. Kitchener H, Swart AMC, Qian W, Amos C, Parmer MKB. Efficacy of systematic pelvic lymphadenectomy in endometrial cancer (MRC ASTEC trial): a randomised study. (vol 373, pg 125, 2009). Lancet. May $232009 ; 373(9677): 1764-1764$.

10. Bogani G, Ditto A, Chiappa V, Raspagliesi F. Sentinel node mapping in endometrial cancer. Transl Cancer Res. Oct 2019;8(6):2218-2219.

11. Schiavone MB, Scelzo C, Straight C, et al. Survival of Patients with Serous Uterine Carcinoma Undergoing Sentinel Lymph Node Mapping. Ann Surg Oncol. Jul 2017;24(7):1965-1971.

12. Baiocchi G, Faloppa CC, Mantoan H, et al. Para-aortic lymphadenectomy can be omitted in most endometrial cancer patients at risk of lymph node metastasis. J Surg Oncol. Aug 1 2017;116(2):220-226.

13. Cusano E, Myers V, Samant R, et al. Prognostic Significance of Lymphovascular Space Invasion in the Absence of Lymph Node Metastases in Early-Stage Endometrial Cancer. Int J Gynecol Cancer. Jun 2018;28(5):890-894.

14. Bosse T, Peters EEM, Creutzberg CL, et al. Substantial lymph-vascular space invasion (LVSI) is a significant risk factor for recurrence in endometrial cancer - A pooled analysis of PORTEC 1 and 2 trials. Eur J Cancer. Sep 2015;51(13):1742-1750.

15. de Boer SM, Powell ME, Mileshkin L, et al. Adjuvant chemoradiotherapy versus radiotherapy alone for women with high-risk endometrial cancer (PORTEC-3): final results of an international, open-label, multicentre, randomised, phase 3 trial. Lancet Oncol.Mar 2018;19(3):295-309.

16. McMillia N, Motter A. NCCN Clinical Practice Guidelines in Oncology of Uterine Neoplasms 2020 v1. 2020;www.nccn.org. Accessed May 1, 2020.

17. Colombo N, Creutzberg C, Amant F, et al. ESMO-ESGO-ESTRO Consensus Conference on Endometrial Cancer Diagnosis, Treatment and Follow-up. Int J Gynecol Cancer. Jan 2016;26(1):2-30.

18. Takeuchi M, Matsuzaki K, Harada M. Evaluating Myometrial Invasion in Endometrial Cancer: Comparison of Reduced Field-of-view Diffusion-weighted Imaging and Dynamic Contrast-enhanced MR Imaging.Magn Reson Med Sci. 2018;17(1):28-34.

19. Peters EEM, Bartosch C, McCluggage WG, et al. Reproducibility of lymphovascular space invasion (LVSI) assessment in endometrial cancer. Histopathology. 2019;75(1):128-136. 
20. Stålberg K, Bjurberg M, Borgfeldt C, et al. Lymphovascular space invasion as a predictive factor for lymph node metastases and survival in endometrioid endometrial cancer - a Swedish Gynecologic Cancer Group (SweGCG) study. Acta oncologica (Stockholm, Sweden).2019;58(11):1628-1633.

21. Boothe D, Wolfson A, Christensen M, Francis S, Werner TL, Gaffney DK. Lymphovascular Invasion in Endometrial Cancer: Prognostic Value and Implications on Adjuvant Radiation Therapy Use. Am J Clin Oncol. 2019;42(7):549-554.

\section{Hosted file}

Tables and Figures.docx available at https://authorea.com/users/342299/articles/469110evaluation-of-factors-influencing-lymph-node-metastasis-in-grade-3-endometrial-cancersa-retrospective-study 